\title{
Neighborhoods of certain $p$-valent analytic functions defined by using generalized differential operators
}

\author{
A. Amourah ${ }^{\mathrm{a}, *}$, O. Dalalaa ${ }^{\mathrm{a}}$, A. Alelaumi ${ }^{\mathrm{a}}$ \\ ${ }^{a}$ Department of Mathematics, Faculty of Science and Technology, Irbid National University, Irbid, Jordan.
}

\begin{abstract}
In this paper, by use of the familiar concept of neighborhoods of $p$-valently analytic functions, the authors show several inclusion relations associated with the $(n, \tau)$-neighborhood of certain subclasses of analytic functions.
\end{abstract}

Keywords: Analytic functions, $(n, \tau)$-neighborhood, Starlike, Convex.

2010 MSC: 30C45.

\section{Introduction and preliminaries}

Let $\mathcal{A}(p)$ denote the class of functions $\mathrm{f}$ of the form:

$$
\mathrm{f}(z)=z^{\mathrm{p}}+\sum_{\mathrm{n}=\mathrm{p}+1}^{\infty} \mathrm{a}_{\mathrm{n}} z^{\mathrm{n}} \quad(z \in \mathbb{U}, \mathrm{p}, \mathrm{n} \in \mathbb{N}=\{1,2,3, \cdots\})
$$

which are analytic and univalent in the open unit disc $\mathbb{U}=\{z \in \mathbb{C}:|z|<1\}$.

The function $f(z)$ in $\mathcal{A}(p)$ is said to be $p$-valent starlike functions of order $\alpha$ and univalent convex of order $\alpha$ in $\mathbb{U}$ if it satisfies:

$$
\operatorname{Re}\left\{\frac{z f^{\prime}(z)}{f(z)}\right\}>\alpha(p \in \mathbb{N}, z \in \mathbb{U}, 0 \leqslant \alpha<p)
$$

and

$$
\operatorname{Re}\left\{1+\frac{z f^{\prime \prime}(z)}{f^{\prime}(z)}\right\}>\alpha(p \in \mathbb{N}, z \in \mathbb{U}, 0 \leqslant \alpha<p)
$$

respectively.

For the function $f \in \mathcal{A}(p)$, we define the following new generalized differential operator as

\footnotetext{
*Corresponding author

Email addresses: alaammour@yahoo.com (A. Amourah ), oladalalaa@yahoo.com (O. Dalalaa), olimat_anas@yahoo.com (A. Alelaumi )

doi:10.31559/glm2020.9.1.1
}

Received 24 May 2020 : Accepted 24 Aug 2020 
follows:

$$
\begin{aligned}
A_{\mu, \lambda, p}^{0}(\alpha, \beta, \delta) f(z) & =f(z), \\
A_{\mu, \lambda, p}^{1}(\alpha, \beta, \delta) f(z) & =\left(\frac{\mu+p(1-\beta(\lambda-\alpha))}{\mu+\lambda}\right) f(z)+\left(\frac{\beta(\lambda-\alpha)+(1-p) \delta}{\mu+\lambda}\right) z f^{\prime}(z) \\
& +\frac{\delta}{\mu+\lambda} z f^{\prime \prime}(z),
\end{aligned}
$$

and for $m=1,2,3, \cdots$

generalises various operators as follows.

$$
\begin{aligned}
A_{\mu, \lambda, p}^{m}(\alpha, \beta, \delta) f(z) & =A_{\mu, \lambda, p}^{m}(\alpha, \beta, \delta)\left(A_{\mu, \lambda, p}^{m-1}(\alpha, \beta, \delta) f(z)\right) . \\
& =z^{p}+\sum_{n=p+1}^{\infty}\left[1+\frac{(n-p)[(\lambda-\alpha) \beta+n \delta]}{\mu+\lambda}\right]^{m} a_{n} z^{n}
\end{aligned}
$$

for $f \in \mathcal{A}(p), \alpha, \delta, \mu \geqslant 0, \beta, \lambda>0, \lambda \neq \alpha, p \in \mathbb{N}, p \neq n$ and $m \in \mathbb{N}_{0}=\mathbb{N} \cup\{0\}$. This generalises various operators as follows.

(i) $A_{\mu, \lambda, 1}^{m}(\alpha, \beta, \delta) f(z)=z+\sum_{n=2}^{\infty}\left[1+\frac{(n-1)[(\lambda-\alpha) \beta+n \delta]}{\mu+\lambda}\right]^{m} a_{n} z^{n}$, the operator introduced and studied by Amourah and Darus (2016) [4].

(ii) $A_{0, \lambda, 1}^{m}(\alpha, \beta, 0) f(z)=z+\sum_{n=2}^{\infty}[1+(n-1)(\lambda-\alpha) \beta]^{m} a_{n} z^{n}$, the operator introduced and studied by Darus and Ibrahim (2009) [12].

(iii) $A_{0, \lambda, 1}^{m}(0,1,0) f(z)=z+\sum_{n=2}^{\infty}[1+(n-1) \lambda]^{m} a_{n} z^{n}$, the operator introduced and studied by Al-Oboudi (2004) [1].

(iv) $A_{0,1,1}^{m}(0,1,0) f(z)=z+\sum_{n=2}^{\infty}[n]^{m} a_{n} z^{n}$, the operator introduced and studied by Sãlãgean (1983) [13]. Further, let $T^{*}(p)$ denoted the supclass of $\mathcal{A}(p)$ consisting functions of the form

$$
f(z)=z^{p}-\sum_{n=p+1}^{\infty} a_{n} z^{n} \quad\left(a_{n} \geqslant 0, n, p \in \mathbb{N}, z \in \mathbb{U}\right) .
$$

Now, we define the $(n, \tau)$ - neighborhood of the function $f \in T^{*}(p)$ by

$$
N_{n, \delta}(f)=\left\{g \in T^{*}(p): g(z)=z^{p}-\sum_{n=p+1}^{\infty}\left|b_{n}\right| z^{n} \text { and } \sum_{n=p+1}^{\infty} n\left|a_{n}-b_{n}\right| \leqslant \tau\right\} .
$$

In particular, for the identity function

$$
h(z)=z^{p}
$$

we immediately have

$$
N_{n, \delta}(h)=\left\{g \in T^{*}(p): g(z)=z^{p}-\sum_{n=p+1}^{\infty} b_{n} z^{n} \text { and } \sum_{n=p+1}^{\infty} n\left|b_{n}\right| \leqslant \tau\right\} .
$$

The concept of the neighborhood was first presented by Goodman [14], and then generalized by Ruscheweyh [15], and applied recently to Neighborhoods of certain $p$-valent analytic functions by Amourah 
and Darus [[3], [9], [2]], and others including Al-Hawary et al. [[10], [4], [6]] and Anakira et al. [[7], [11], [8]] .

We define and study some $(n, \tau)$ - neighborhood properties. The classes $G^{*}(b, p)$ and $G^{*}(b, p, \chi)$ are defined as follows:

Definition 1.1. A function $f \in T^{*}$ is said to be in the class $G^{*}(b, p)$ if and only if

$$
\left|\frac{1}{b}\left[\frac{z\left(A_{\mu, \lambda, p}^{m}(\alpha, \beta, \delta) f(z)\right)^{\prime}}{A_{\mu, \lambda, p}^{m}(\alpha, \beta, \delta) f(z)}-p\right]\right|<\zeta,
$$

for some $\alpha \geqslant 0,0<\zeta \leqslant 1, \beta, \lambda, \mu>0, \lambda \neq \alpha, p \in \mathbb{N}, p \neq n, b \in \mathbb{C}-\{0\}$ and $m \in \mathbb{N}_{0}$ and for all $z \in \mathbb{U}$.

Definition 1.2. A function $f \in T^{*}(p)$ is said to be in the class $G^{*}(b, p, \chi)$ if and only if

$$
\left|\frac{1}{b}\left[(1-\chi) \frac{A_{\mu, \lambda, p}^{m}(\alpha, \beta, \delta) f(z)}{z^{p}}+\chi \frac{\left(A_{\mu, \lambda, p}^{m}(\alpha, \beta, \delta) f(z)\right)^{\prime}}{p z^{p-1}}-1\right]\right|<\zeta,
$$

for some $\alpha, \chi \geqslant 0,0<\zeta \leqslant 1, \beta, \lambda, \mu>0, \lambda \neq \alpha, p \in \mathbb{N}, p \neq n, b \in \mathbb{C}-\{0\}$ and $m \in \mathbb{N}_{0}$ and for all $z \in \mathbb{U}$.

\section{Neighborhoods for the classes $G^{*}(b, p)$ and $G^{*}(b, p, \chi)$}

In our investigation of the inclution relations involving $N_{n, \delta}(h)$, we shall require theorem 2.1 and theorem 2.2 below:

Theorem 2.1. Let the function $\mathrm{f}(z) \in \mathrm{T}^{*}(\mathrm{p})$ be defined by (1.6). Then $\mathrm{f}$ is in the class $\mathrm{G}^{*}(\mathrm{~b}, \mathrm{p})$ if and only if

$$
\sum_{n=p+1}^{\infty}(n-p+\zeta|b|)\left[1+\frac{(n-p)[(\lambda-\alpha) \beta+n \delta]}{\mu+\lambda}\right]^{m} a_{n} \leqslant \zeta|b|,
$$

where $\alpha \geqslant 0,0<\zeta \leqslant 1, \beta, \lambda, \mu>0, \lambda \neq \alpha, p \in \mathbb{N}, p \neq n, b \in \mathbb{C}-\{0\}$ and $m \in \mathbb{N}_{0}$.

Proof. We first suppose that $f \in G^{*}(b, p)$. Then by appealing to the condition (1.10), we readily obtain

$$
\operatorname{Re}\left\{\frac{z\left(A_{\mu, \lambda, p}^{m}(\alpha, \beta, \delta) f(z)\right)^{\prime}}{A_{\mu, \lambda, p}^{m}(\alpha, \beta, \delta) f(z)}-p\right\}>-\zeta|b| .
$$

or, equivalently,

$$
\begin{aligned}
& \operatorname{Re}\left\{\frac{-\sum_{n=p+1}^{\infty}(n-p)\left[1+\frac{(n-p)[(\lambda-\alpha) \beta+n \delta]}{\mu+\lambda}\right]^{m} a_{n} z^{n-p}}{1-\sum_{n=p}^{\infty}\left[1+\frac{(n-p)[(\lambda-\alpha) \beta+n \delta]}{\mu+\lambda}\right]^{m} a_{n} z^{n-p}}\right\} \\
& >-\zeta|b| .
\end{aligned}
$$

We now choose values of $z$ on the real axis and let $z \rightarrow 1^{-}$through real values. Then the inequality (2.2) immediately yields the desired condition (2.1).

Conversely, by applying the hypothesis (2.1) and letting $|z|=1$, we find from (1.10) that 


$$
\begin{aligned}
& \left|\frac{z\left(A_{\mu, \lambda, p}^{m}(\alpha, \beta, \delta) f(z)\right)^{\prime}}{A_{\mu, \lambda, p}^{m}(\alpha, \beta, \delta) f(z)}-p\right| \\
& =\left|\frac{\sum_{n=p}^{\infty}(n-p)\left[1+\frac{(n-p)[(\lambda-\alpha) \beta+n \delta]}{\mu+\lambda}\right]^{m} a_{n} z^{n-p} \mid}{1-\sum_{n=p}^{\infty}\left[1+\frac{(n-p)[(\lambda-\alpha) \beta+n \delta]}{\mu+\lambda}\right]^{m} a_{n} z^{n-p}}\right| \\
& \left.\leqslant \frac{n=p+1}{1-\sum_{n=p}^{\infty}\left[1+\frac{(n-p)[(\lambda-\alpha) \beta+n \delta]}{\mu+\lambda}\right]^{m} a_{n}}\right]^{m} a_{n} \\
& \zeta|b|\left(1-\sum_{n=p}^{\infty}\left[1+\frac{(n-p)[(\lambda-\alpha) \beta+n \delta]}{\mu+\lambda}\right]^{m} a_{n}\right) \\
& \leqslant \frac{1-\sum_{n=p}^{\infty}\left[1+\frac{(n-p)[(\lambda-\alpha) \beta+n \delta]}{\mu+\lambda}\right]^{m} a_{n}}{\mu+\lambda) \beta+n}\left[1+\frac{(n-p)[(\lambda-\alpha)}{m}[|b| .\right.
\end{aligned}
$$

This implies

$$
\sum_{n=p+1}^{\infty}(n-p+\zeta|b|)\left[1+\frac{(n-p)[(\lambda-\alpha) \beta+n \delta]}{\mu+\lambda}\right]^{m} a_{n} \leqslant \zeta|b| .
$$

Then, we have $f \in G^{*}(b, p)$.

Similarly, we can prove the following result:

Theorem 2.2. Let the function $\mathrm{f}(z) \in \mathrm{T}^{*}(\mathrm{p})$ be defined by (1.6). Then $\mathrm{f}$ is in the class $\mathrm{G}^{*}(\mathrm{~b}, \mathrm{p}, \mathrm{x})$ if and only if

$$
\sum_{n=p+1}^{\infty}(p+\chi(n-p))\left[1+\frac{(n-p)[(\lambda-\alpha) \beta+n \delta]}{\mu+\lambda}\right]^{m} a_{n} \leqslant p \zeta|b| .
$$

Proof. We first suppose that $f \in G^{*}(b, p, \chi)$. Then by appealing to the condition (1.11), we readily obtain

$$
\operatorname{Re}\left\{(1-\chi) \frac{A_{\mu, \lambda, p}^{m}(\alpha, \beta, \delta) f(z)}{z^{p}}+\chi \frac{\left(A_{\mu, \lambda, p}^{m}(\alpha, \beta, \delta) f(z)\right)^{\prime}}{p z^{p-1}}-1\right\}>-\zeta|b|
$$

or, equivalently

$$
\begin{aligned}
& \operatorname{Re}\left\{-\sum_{n=p+1}^{\infty}\left(1-\chi+\frac{n}{p} \chi\right)\left[1+\frac{(n-p)[(\lambda-\alpha) \beta+n \delta]}{\mu+\lambda}\right]^{m} a_{n} z^{n-p}\right\} \\
& >-\zeta|b|,
\end{aligned}
$$

We now choose values of $z$ on the real axis and let $z \rightarrow 1$ through real values. Then the inequality (2.8) immediately yields the desired condition (2.7). 
Conversely, by applying the hypothesis (2.7) and letting $z=1$, we find from (1.11) that

$$
\begin{aligned}
& \left|(1-\chi) \frac{A_{\mu, \lambda, p}^{m}(\alpha, \beta, \delta) f(z)}{z^{p}}+\chi \frac{\left(A_{\mu, \lambda, p}^{m}(\alpha, \beta, \delta) f(z)\right)^{\prime}}{p z^{p-1}}-1\right| \\
& =\left|\sum_{n=p+1}^{\infty}\left(1-\chi+\frac{n}{p} \chi\right)\left[1+\frac{(n-p)[(\lambda-\alpha) \beta+n \delta]}{\mu+\lambda}\right]^{m} a_{n} z^{n-p}\right| \\
& \leqslant \sum_{n=p+1}^{\infty}\left(1-\chi+\frac{n}{p} \chi\right)\left[1+\frac{(n-p)[(\lambda-\alpha) \beta+n \delta]}{\mu+\lambda}\right]^{m} a_{n}\left|z^{n-p}\right| \\
& \leqslant \sum_{n=p+1}^{\infty}\left(1-\chi+\frac{n}{p} \chi\right)\left[1+\frac{(n-p)[(\lambda-\alpha) \beta+n \delta]}{\mu+\lambda}\right]^{m} a_{n}<\zeta|b| .
\end{aligned}
$$

Thus, we have

$$
\sum_{n=2}^{\infty}(p+\chi(n-p))\left[1+\frac{(n-p)[(\lambda-\alpha) \beta+n \delta]}{\mu+\lambda}\right]^{m} a_{n} \leqslant p \zeta|b| .
$$

Hence, we have $f \in G^{*}(b, p, \chi)$.

Our first inclusion relation involving $N_{k, \delta}(h)$ is given by theorem 2.3 below.

Theorem 2.3. If

$$
\tau=\frac{\zeta|b|(1+p)}{(1+\zeta|b|)\left[1+\frac{(n-p)[(\lambda-\alpha) \beta+n \delta]}{\mu+\lambda}\right]^{m}} \quad(|b|<1),
$$

then

$$
\mathrm{G}^{*}(b, p) \subset \mathrm{N}_{n, \delta}(\mathrm{h}) .
$$

Proof. Let $f(z) \in \mathrm{G}^{*}(b, p)$. Then, in view of the assertion (2.1) of theorem 2.1, we have

$$
\begin{gathered}
(1+\zeta|b|)\left[1+\frac{[(\lambda-\alpha) \beta+n \delta]}{\mu+\lambda}\right]^{m} \sum_{n=p+1}^{\infty} a_{n} \leqslant \\
\sum_{n=p+1}^{\infty}(n-p+\zeta|b|)\left[1+\frac{(n-p)[(\lambda-\alpha) \beta+n \delta]}{\mu+\lambda}\right]^{m} a_{n} \leqslant \zeta|b|,
\end{gathered}
$$

which readily yeilds

$$
\sum_{n=p+1}^{\infty} a_{n} \leqslant \frac{\zeta|b|}{(1+\zeta|b|)\left[1+\frac{[(\lambda-\alpha) \beta+n \delta]}{\mu+\lambda}\right]^{m}}
$$

Making use of (2.1) again, in conjunction with (2.11), we get

$$
\begin{aligned}
{\left[1+\frac{[(\lambda-\alpha) \beta+n \delta]}{\mu+\lambda}\right]^{m} \sum_{n=p+1}^{\infty} n a_{n} } & \leqslant \zeta|b|+\left[1+\frac{[(\lambda-\alpha) \beta+n \delta]}{\mu+\lambda}\right]^{m}(1-\zeta|b|) \sum_{n=p+1}^{\infty} a_{n} \\
& \leqslant \frac{\zeta|b|(1+p)}{(1+\zeta|b|)} .
\end{aligned}
$$

Hence

$$
\sum_{n=p+1}^{\infty} n a_{n} \leqslant \frac{\zeta|b|(1+p)}{(1+\zeta|b|)\left[1+\frac{[(\lambda-\alpha) \beta+n \delta]}{\mu+\lambda}\right]^{m}}=\tau, \quad(|b|<1)
$$

which, by means of the definition (1.9), establishes the inclusion (2.10) asserted by theorem 2.3. Similarly, by applying theorem 2.2 instead of theorem 2.1, we now prove theorem 2.4 below. 
Theorem 2.4. If

$$
\tau=\frac{p \zeta|b|(1+p)}{(p+\chi)\left[1+\frac{[(\lambda-\alpha) \beta+n \delta]}{\mu+\lambda}\right]^{m}} \quad(|b|<p),
$$

then

$$
\mathrm{G}^{*}(b, \mathrm{p}, \chi) \subset \mathrm{N}_{\mathrm{n}, \delta}(\mathrm{h}) .
$$

Proof. Let $f(z) \in \mathrm{G}^{*}(b, p, \chi)$. Then, in view of the assertion (2.7) of theorem 2.2, we have

$$
\begin{gathered}
(p+\chi)\left[1+\frac{[(\lambda-\alpha) \beta+n \delta]}{\mu+\lambda}\right]^{m} \sum_{n=p+1}^{\infty} a_{n} \leqslant \\
\sum_{n=p+1}^{\infty}(p+\chi(n-p))\left[1+\frac{(n-p)[(\lambda-\alpha) \beta+n \delta]}{\mu+\lambda}\right]^{m} a_{n} \leqslant p \zeta|b|
\end{gathered}
$$

which readily yeilds

$$
\sum_{n=p+1}^{\infty} a_{n} \leqslant \frac{p \zeta|b|}{(p+\chi)\left[1+\frac{[(\lambda-\alpha) \beta+n \delta]}{\mu+\lambda}\right]^{m}} .
$$

Making use of (2.7) again, in conjunction with (2.13), we get

$$
\begin{aligned}
\chi\left[1+\frac{[(\lambda-\alpha) \beta+n \delta]}{\mu+\lambda}\right]^{m} \sum_{n=p+1}^{\infty} n a_{n} & \leqslant p \zeta|b|+p(x-1)\left[1+\frac{[(\lambda-\alpha) \beta+n \delta]}{\mu+\lambda}\right]^{m} \sum_{n=p+1}^{\infty} a_{n} \\
& \leqslant \frac{p \zeta|b|(p+1) \chi}{(p+x)} .
\end{aligned}
$$

Hence

$$
\sum_{n=p+1}^{\infty} n a_{n} \leqslant \frac{p \zeta|b|(p+1)}{(p+\chi)\left[1+\frac{[(\lambda-\alpha) \beta+n \delta]}{\mu+\lambda}\right]^{m}}=\tau, \quad(|b|<p)
$$

which, by means of the definition (1.9), establishes the inclusion (2.12) asserted by theorem 2.4.

\section{Neighborhoods for the classes $G_{\varepsilon}^{*}(b, p)$ and $G_{\varepsilon}^{*}(b, p, \chi)$}

In this section, we determine the neighborhood for the each classes $G_{\varepsilon}^{*}(b, p)$ and $G_{\varepsilon}^{*}(b, p, \chi)$, which we define as follows. A function $\mathrm{f}(z) \in \mathcal{A}(\mathrm{p})$ is said to be in the class $\mathrm{G}_{\mathcal{\varepsilon}}^{*}(\mathrm{~b}, \mathrm{p})$ if there exists a function $\mathrm{g}(z) \in$ $\mathrm{G}_{\varepsilon}^{*}(b, p)$ such that

$$
\left|\frac{f(z)}{g(z)}-1\right|<p-\varepsilon,(0 \leqslant \varepsilon<p) .
$$

Analogously, a function $f(z) \in \mathcal{A}(p)$ is said to be in the class $G_{\mathcal{E}}^{*}(b, p, \chi)$ if there exists a function $g(z) \in$ $\mathrm{G}_{\varepsilon}^{*}(b, p, \chi)$ such that the inequality (3.1) holds true.

Theorem 3.1. If $\mathrm{g}(z) \in \mathrm{G}_{\varepsilon}^{*}(\mathrm{~b}, \mathrm{p})$ and

$$
\varepsilon=1-\frac{\tau(1+\zeta|b|)\left[1+\frac{[(\lambda-\alpha) \beta+n \delta]}{\mu+\lambda}\right]^{m}}{(p+1)(1+\zeta|b|)\left[1+\frac{[(\lambda-\alpha) \beta+n \delta]}{\mu+\lambda}\right]^{m}-\zeta|b|},
$$

then

$$
\mathrm{N}_{\mathrm{n}, \delta}(\mathrm{g}) \subset \mathrm{G}_{\varepsilon}^{*}(\mathrm{~b}, \mathrm{p}) .
$$


Proof. Suppose that $f(z) \in N_{n, \delta}(g)$. We find from (1.7) that

$$
\sum_{n=p+1}^{\infty} n\left|a_{n}-b_{n}\right| \leqslant \tau
$$

which readily implies that

$$
\sum_{n=p+1}^{\infty}\left|a_{n}-b_{n}\right| \leqslant \frac{\tau}{p+1} .
$$

Next, since $g(z) \in G^{*}(b, p)$, we have [cf. equation 2.11]

$$
\sum_{n=p+1}^{\infty} b_{n} \leqslant \frac{\zeta|b|}{(1+\zeta|b|)\left[1+\frac{[(\lambda-\alpha) \beta+n \delta]}{\mu+\lambda}\right]^{m}}
$$

letting $|z| \rightarrow 1$, so we have

$$
\begin{aligned}
& \left|\frac{f(z)}{g(z)}-1\right| \leqslant \frac{\sum_{n=p}^{\infty}\left|a_{n}-b_{n}\right|}{1-\sum_{n=p}^{\infty}\left|b_{n}\right|} \leqslant \frac{\tau}{p+1} \cdot \frac{(1+\zeta|b|)\left[1+\frac{[(\lambda-\alpha) \beta+n \delta]}{\mu+\lambda}\right]^{m}}{(1+\zeta|b|)\left[1+\frac{[(\lambda-\alpha) \beta+n \delta]}{\mu+\lambda}\right]^{m}-\zeta|b|} \\
& =p-\varepsilon
\end{aligned}
$$

provided that $\alpha$ is given by (3.2). Thus, by the above defnition, $f(z) \in G_{\varepsilon}^{*}(b, p)$ for $\varepsilon$ given by (3.2).

This evidently proves theorem 3.1.

Our proof of theorem 3.2 below is much akin to that of theorem 3.1.

Theorem 3.2. If $\mathrm{g}(z) \in \mathrm{G}_{\mathcal{\varepsilon}}^{*}(\mathrm{~b}, \mathrm{p}, \mathrm{\chi})$ and

$$
\varepsilon=p-\frac{\tau(p+\chi)\left[1+\frac{[(\lambda-\alpha) \beta+n \delta]}{\mu+\lambda}\right]^{m}}{(p+1)(p+\chi)\left[1+\frac{[(\lambda-\alpha) \beta+n \delta]}{\mu+\lambda}\right]^{m}-p \zeta|b|},
$$

then

$$
\mathrm{N}_{\mathrm{n}, \delta}(\mathrm{g}) \subset \mathrm{G}_{\varepsilon}^{*}(\mathrm{~b}, \mathrm{p}, \chi) .
$$

Proof. Suppose that $f(z) \in N_{n, \delta}(g)$. We find from (1.7) that

$$
\sum_{n=p+1}^{\infty} n\left|a_{n}-b_{n}\right| \leqslant \tau
$$

which readily implies that

$$
\sum_{n=p+1}^{\infty}\left|a_{k}-b_{k}\right| \leqslant \frac{\tau}{p+1} .
$$

Next, since $g(z) \in G_{\mathcal{\varepsilon}}^{*}(b, p, \chi)$, we have [cf. equation 2.13]

$$
\sum_{n=p+1}^{\infty} b_{n} \leqslant \frac{p \zeta|b|}{(p+\chi)\left[1+\frac{[(\lambda-\alpha) \beta+n \delta]}{\mu+\lambda}\right]^{m}}
$$


letting $|z| \rightarrow 1$, so we have

$$
\begin{aligned}
& \left|\frac{f(z)}{g(z)}-1\right| \leqslant \frac{\sum_{n=p}^{\infty}\left|a_{n}-b_{n}\right|}{1-\sum_{n=p}^{\infty}\left|b_{n}\right|} \leqslant \frac{\delta}{p+1} \cdot \frac{(p+\chi)\left[1+\frac{[(\lambda-\alpha) \beta+n \delta]}{\mu+\lambda}\right]^{m}}{(p+\chi)\left[1+\frac{[(\lambda-\alpha) \beta+n \delta]}{\mu+\lambda}\right]^{m}-p \zeta|b|} \\
& =p-\varepsilon
\end{aligned}
$$

provided that $\varepsilon$ is given by (3.5). Thus, by the above defnition, $f(z) \in G_{\varepsilon}^{*}(b, p, \chi)$ for $\varepsilon$ given by (3.5).

This evidently completes our proof of theorem 3.2.

\section{References}

[1] F. Al-Oboudi, On univalent functions defined by a generalized Salagean operator, International Journal of Mathematics and Mathematical Sciences. 2004. 27(2004), 1429-36. 1

[2] A. Amourah and Feras Yousef, Some properties of a class of analytic functions involving a new generalized differential operator, Boletim da Sociedade Paranaense de Matematica 38.6 (2019), 33-42. https://doi.org/10.5269/bspm.v38i6.40530 1

[3] A. Amourah, Tariq Al-Hawary and Maslina Darus, Neighborhoods of certain $\mathrm{p}$-valent analytic functions defined by a generalized differential operator, International Information Institute (Tokyo). Information 19.9B (2016), 3943. 1

[4] A. Amourah, and Maslina Darus, Some properties of a new class of univalent functions involving a new generalized differential operator with negative coefficients, Indian Journal of Science and Technology 9.36 (2016), 1-7. https:/ / doi.org/10.17485/ijst/2016/v9i36/97738 1, 1

[5] A. Amourah, Initial bounds for analytic and bi-univalent functions by means of (p,q)-Chebyshev polynomials defined by differential operator, General Letters in Mathematics 7. 2 (2019), 45-51. https:/ / doi.org/10.31559/glm2019.7.2.1 1

[6] A. Amourah, Initial bounds for analytic and bi-univalent functions by means of ( $p, q)$-Chebyshev polynomials defined by differential operator, General Letters in Mathematics 7. 2 (2019), 45-51. https://doi.org/10.31559/glm2019.7.2.1 1

[7] N. Anakira, A. Alomari, A. F. Jameel and I. Hashim, Multistage optimal homotopy asymptotic method for solving initial-value problems, Journal of Nonlinear Science and Applications 9.4 (2016), 1826-1843. https:/ / doi.org/10.22436/jnsa.009.04.37 1

[8] N. Anakira, A. F. Jameel, A. K. Alomari, Azizan Saaban, Mohammad Almahameed and Ishak Hashim, Approximate solutions of multi-pantograph type delay differential equations using multistage optimal homotopy asymptotic method, Journal of Mathematical and Fundamental Sciences 50. 3 (2018). 221-232. 1

[9] M. Darus and R.Ibrahim, On subclasses for generalized operators of complex order, Far East Journal of Mathematical Sciences 33. 3(2009), 299-308. 1

[10] A. W. Goodman, Univalent functions and nonanalytic curves, Proceedings of the American Mathematical Society 8. 3(1957), 598-601. https://doi.org/10.1090/s0002-9939-1957-0086879-9 1

[11] A. F. Jameel, N. Anakira, A. K. Alomari, Ishak Hashim, and Shaher Momani, A New Approximation Method for Solving Fuzzy Heat Equations, Journal of Computational and Theoretical Nanoscience 13. 11 (2016), 7825-7832. https://doi.org/10.1166/jctn.2016.5784 1

[12] S. Ruscheweyh, Neighborhoods of univalent functions, Proceedings of the American Mathematical Society 81. 4(1981), 521-527. https: / / doi.org/10.1090/s0002-9939-1981-0601721-6 1

[13] G. Salagean, Subclasses of univalent functions. Complex Analysis-Fifth Romanian-Finnish Seminar, Springer Berlin Heidelberg; 1983. p. 362-72. 1

[14] Tariq Al-Hawary, A. A. Amourah and Laith Abualigah. Neighborhoods of a class of analytic functions with negative coefficients associated with Jacksons-(p,q) derivative, International Journal 8.6 (2019). https://doi.org/10.30534/ijsait/2019 1

[15] Tariq Al-Hawary, A. A. Amourah and Maslina Darus, Differential sandwich theorems for $\mathrm{p}$-valent functions associated with two generalized differential operator and integral operator, International Information Institute (Tokyo). Information 17. 8(2014), 3559. 1 